\title{
Ureteroscopy for Symptomatic Hydrocalices: A Case Series
}

\author{
KATHLEEN KIERAN, M.D., CALEB P. NELSON, M.D., and J. STUART WOLF, JR., M.D.
}

\begin{abstract}
Background: Hydrocalix is an uncommon condition that typically is treated with percutaneous dilation of the stenotic infundibulum. Ureteroscopy has the advantage of minimizing invasiveness.

Methods: We reviewed our experience with 12 retrograde ureteroscopies for hydrocalix in 10 patients.

Results: Access to the hydrocalix could not be achieved in one. Of the remaining 11, the original infundibulum was dilated in all but one, in whom neoinfundibulotomy was performed with a laser. The laser was used in eight cases, balloon dilation with cautery in two, and cautery alone in one. Of the seven hydrocalices bearing stones, four were rendered stone free. Immediate symptomatic success was achieved after 6 of the 11 evaluable procedures (one patient was lost to follow-up after a technically successful procedure). Of the five symptomatic failures, three went on to additional treatment (one ureteroscopy and two percutaneous), one elected against treatment despite recurrence of stenosis, and one had persistent pain owing to stones even though the hydrocalix was cured. Of the six symptomatic successes, three were without symptoms at a mean 25 months of follow-up, two have had recurrences necessitating additional treatment (both percutaneous) a mean of 51 months later, and one did not have adequate imaging follow-up.

Conclusion: Ureteroscopy for hydrocalix achieved technical success in most patients, but relief of symptoms followed only about half of the procedures. A trial of ureteroscopy does not preclude subsequent success with a percutaneous approach. Ureteroscopy is a reasonable option for hydrocalix for patients who wish to avoid percutaneous surgery.
\end{abstract}

\section{INTRODUCTION}

$\mathbf{H}$ YDROCALIX ARISES when a renal calix is obstructed by a narrowed infundibulum. The presence of a renal papilla together with a stenotic outflow tract defines a hydrocalix, distinguishing it from other focal upper-tract dilations such as caliceal diverticula (intrarenal collecting system outpouchings without a renal papilla) and megacalicosis (idiopathic caliceal dilatation without infundibular narrowing). Hydrocalices may be congenital or acquired secondary to infection, iatrogenic injury, or trauma. ${ }^{1,2}$ Urine production continues in the hydrocalix, which contributes to the presenting symptoms of hematuria, urinary-tract infection, or pain. Associated nephrolithiasis may reflect underlying metabolic abnormalities as well as urinary stasis. ${ }^{3}$

Treatment of a hydrocalix involves decompression of the distended calix and relief of intrinsic or extrinsic obstruction, along with treatment of coexisting nephrolithiasis. Open renal exploration is no longer performed frequently, although partial nephrectomy (open surgical or laparoscopic) may be warranted if the overlying parenchyma is atrophic. The most commonly reported procedure is percutaneous dilation of the stenotic infundibulum with removal of associated renal calculi. ${ }^{4-7} \mathrm{We}$ are aware of only one prior case report of ureteroscopy for hydrocalix ${ }^{8}$ and a mention of the possibility of such treatment in one other publication. ${ }^{9}$

Ureteroscopy is the procedure of choice at many institutions for stones not amenable to shockwave lithotripsy (SWL) unless a large stone burden or other consideration prompts percutaneous nephrolithotomy. Skill in flexible ureteroscopy has now been acquired during training by a generation of urologists. Given the improved postoperative recovery profile and potential cost savings associated with the decreased resource utilization of outpatient ureteroscopy, this technique should be considered for the treatment of additional renal pathology, such as hydrocalix. We report a case series of 12 endoscopic treatments in 10 patients for relief of symptoms related to hydrocalix.

Department of Urology, University of Michigan, Ann Arbor, Michigan. 


\section{PATIENTS AND METHODS}

Between December 1999 and May 2003, nine patients at the University of Michigan Health System and one at the Ann Arbor Veteran's Administration Medical Center underwent a total of 12 ureteroscopic procedures for hydrocalix (Table 1). All were symptomatic, nine with flank pain and one with a fungal urinary-tract infection. The etiology of the infundibular stenosis was unknown in most cases. Patient 5 had developed multiple hydrocalices after receiving radiation to the kidney for Wilms' tumor and has been the subject of a prior report. ${ }^{10}$

Ureteroscopy was performed using standard (single active deflection) $6.9 \mathrm{~F}$ or $7.5 \mathrm{~F}$ flexible ureteroscopes. Under fluoroscopic and visual guidance, the stenosed infundibulum was accessed, and any stones identified were treated with laser lithotripsy or basket extraction. Infundibular stenosis was treated with a $200-\mu \mathrm{m}$ or $300-\mu \mathrm{m}$ holmium:YAG laser at 8 to $10 \mathrm{~W}$ and 10 to $12 \mathrm{~Hz}$ or Bugbee electrocautery with or without balloon dilatation. Neoinfundibulotomy was performed if the stenosed infundibulum could not be seen or accessed. Confirmation of resolution of stenosis was obtained by fluoroscopic assessment of the caliber of the infundibulum after retrograde injection of contrast and by visual inspection.

A ureteral stent was placed if there was concern about limited drainage of the collecting system. All patients were discharged to home on the day of surgery with oral pain medications and a short course of oral antibiotics. Patients generally were seen 2 to 4 weeks postoperatively to evaluate symptomatic improvement and variably thereafter depending on other circumstances. Postoperative imaging included ultrasonography and abdominal radiography, intravenous urography, or CT.

\section{RESULTS}

All but one procedure involved treatment of a solitary infundibular stenosis; patient 5 underwent treatment of four infundibular stenoses at her first procedure. Eight patients had one procedure, and two patients ( 5 and 8 ) had two; the second procedure in patient 5 was on a second hydrocalix rather than a recurrence.

Endoscopic access and dilation of the infundibulum was technically successful in all but one patient (No. 7), in whom a lower-pole calix could not be accessed. Subsequent percutaneous access with infundibular dilatation was successful in this patient. Caliceal access was successful but lithotripsy incomplete in one patient (No. 2) secondary to inability to manipulate the ureteroscope widely in the infundibulum, and in two other patients (Nos. 4 and 6), stones were thought to have been removed completely, but there were residual fragments in the treated calix on follow-up imaging. The infundibular stenosis was opened using a Ho:YAG laser in eight cases, combined balloon dilatation and electrocautery in two cases, and by electrocautery alone in one. In one patient (No. 6), the infundibulum of the involved calix could not be found, and a neoinfundibulotomy was created using the Ho:YAG laser under fluoroscopic and visual guidance. Stones were identified in seven procedures, and follow-up imaging confirmed stone-free status in the treated area in four.

Only one perioperative complication occurred. Patient 6 had recurrent left-flank pain 2 days postoperatively. Imaging suggested ureteral obstruction, likely from edema; stent placement for 6 days relieved her pain. Of note, this procedure was the one case that required neoinfundibulotomy and one of only three that did not involve a postoperative stent.

The outcomes are listed in Table 2. Of the 10 patients, one (No. 3) was lost to follow-up after a technically successful procedure. Endoscopic treatment of the stenotic hydrocalix was technically successful in 10 initial procedures $(90.9 \%)$. Of the 10 successful procedures, symptoms were relieved in 6 of 9 patients $(66.7 \%)$, excluding the patient lost to follow-up. Stenosis recurrence was observed after 4 of 8 procedures $(50 \%)$ in patients with adequate postoperative imaging. Three of the five patients with symptomatic failure went on to early additional treatment (a second attempt at ureteroscopy in patient 8, percutaneous dilation in patient 7 , and percutaneous treatment after the second ureteroscopy in patient 8). Patient 10 elected against treatment despite documented recurrence of stenosis at

Table 1. Patient Demographics and Treatment Techniques

\begin{tabular}{|c|c|c|c|c|c|c|c|}
\hline$P t$. & Age/sex & Side & Region & Technique & Stone? & Access? & Stent? \\
\hline 1 & $35 \mathrm{M}$ & Left & Lower & Laser & Yes & Yes & Yes \\
\hline 2 & $57 \mathrm{M}$ & Left & Lower & Laser & Yes & Yes & Yes \\
\hline 3 & $34 \mathrm{~F}$ & Right & Lower & Laser & Yes & Yes & Yes \\
\hline 4 & $66 \mathrm{M}$ & Right & Middle & Cautery & Yes & Yes & Yes \\
\hline 5 & $26 \mathrm{~F}$ & Left & $\begin{array}{l}\text { Upper, } \\
\text { lower }\end{array}$ & $\begin{array}{l}\text { Balloon } \\
\quad(5 \mathrm{~mm}), \\
\text { cautery }\end{array}$ & Yes & Yes & Yes \\
\hline 5 & $27 \mathrm{~F}$ & Left & Upper & $\begin{array}{l}\text { Balloon } \\
\quad(4 \mathrm{~mm}), \\
\text { cautery }\end{array}$ & No & Yes & Yes \\
\hline 6 & $48 \mathrm{~F}$ & Left & Upper & Laser & Yes & Yes & No \\
\hline 7 & $51 \mathrm{~F}$ & Right & Lower & NA & No & No & No \\
\hline 8 & $48 \mathrm{~F}$ & Left & Middle & Laser & No & Yes & Yes \\
\hline 8 & $49 \mathrm{~F}$ & Left & Middle & Laser & No & Yes & Yes \\
\hline 9 & $59 \mathrm{~F}$ & Right & Middle & Laser & Yes & Yes & Yes \\
\hline 10 & $43 \mathrm{~F}$ & Left & Upper & Laser & No & Yes & No \\
\hline
\end{tabular}


Table 2. Outcomes of Procedures

\begin{tabular}{|c|c|c|c|c|c|c|}
\hline Patient & $\begin{array}{l}\text { Success, } \\
\text { stone? }\end{array}$ & $\begin{array}{l}\text { Success, } \\
\text { stenosis? }\end{array}$ & $\begin{array}{l}\text { Success, } \\
\text { symptoms? }\end{array}$ & $\begin{array}{c}\text { Stenosis } \\
\text { recurrence? }\end{array}$ & $\begin{array}{l}\text { Follow-up } \\
\text { (mos) }\end{array}$ & $\begin{array}{c}\text { Additional } \\
\text { treatment }\end{array}$ \\
\hline 1 & Yes & Yes & Yes & No & 66 & None \\
\hline 2 & No & Yes & No & No & 13 & None \\
\hline 3 & Yes & Yes & $?$ & $?$ & Lost to follow-up & - \\
\hline 4 & No & Yes & Yes & $?$ & 1 & None $^{\mathrm{a}}$ \\
\hline 5 & Yes & Yes & Yes & No & 8 & None \\
\hline 5 & - & Yes & Yes & Yes & 3 & Percutaneous \\
\hline 6 & No & Yes & Yes & Yes & 55 & Percutaneous \\
\hline 7 & - & No & No & - & - & Percutaneous \\
\hline 8 & - & Yes & No & Yes & 2 & Repeat URS \\
\hline 8 & - & Yes & No & Yes & 2 & Percutaneous \\
\hline 9 & Yes & Yes & Yes & No & 1.5 & None \\
\hline 10 & - & Yes & No & Yes & 1 & None \\
\hline
\end{tabular}

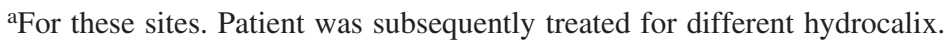

1 month. Patient 2 later underwent treatment for persistent stones after successful treatment of the hydrocalix (13 months' follow-up). Of the six symptomatic successes, patient 4 did not have adequate imaging at the 1-month follow-up visit.

Three patients (Nos. 1, first procedure in 5, and 9) were without symptoms or recurrence of stenosis at a mean 25 months' follow-up. Early infundibular stenosis recurrence was seen in two patients (Nos. 8 and 10) and late recurrence in two patients several years after initial treatment (Nos. 5 and 6); all were managed percutaneously. Patient 5 developed recurrent flank pain 47 months after her second procedure; CT imaging demonstrated a poorly draining upper-pole calix, although this was thought to be a new rather than a recurrent hydrocalix. Retrograde ureteroscopy was unsuccessful in identifying the involved calix, and so antegrade stone extraction and infundibular dilatation were performed after percutaneous access was accomplished. A CT scan 6 months after the procedure demonstrated excellent drainage of the treated calix. Patient 6 had recurrent flank pain 55 months after ureteroscopic treatment of a hydrocalix; ureteroscopic access was attempted without success by a community urologist, and she was subsequently referred back to our institution, where stone extraction and dilatation of the neck of the hydrocalix were performed antegrade via a percutaneous nephrolithotomy. A CT urogram 3 months after the percutaneous procedure demonstrated excellent patency of the previously obstructed infundibulum.

\section{DISCUSSION}

Successful treatment of hydrocalices and caliceal diverticula entails not only improved urinary drainage but also treatment of associated conditions such as extrinsic compression or, more commonly, stones. Thus, at least two distinct components of the procedure are necessary in most patients to achieve radiographic success and symptomatic relief.

To our knowledge, there is only one prior case report of ureteroscopy for hydrocalix. ${ }^{8}$ Kim and Gerber used a Ho:YAG laser and 7-mm balloon to widen a stenotic middle-caliceal infundibulum, with an excellent appearance on intravenous urography 6 weeks postoperatively and continued absence of symp- toms 12 weeks after surgery. Also, Chong and associates ${ }^{9}$ mentioned the possibility of such treatment in their review of ureteroscopy for caliceal diverticula. Given this paucity of data, we can best compare our experience with that of the ureteroscopic treatment of caliceal diverticula. One important caveat must be borne in mind when considering this comparison, however. In the treatment of caliceal diverticula, complete restenosis of the neck can still be associated with cure, whereas in the case of hydrocalices, any degree of restenosis would lead to failure because the functioning papillary unit continues to excrete urine into the cavity.

Caliceal diverticula have been treated with SWL, open or percutaneous renal surgery, and, more recently, ureteroscopy. Shockwave lithotripsy enables noninvasive treatment of diverticular renal stones, but fragments may not pass, given the persistent restriction of caliceal drainage. ${ }^{11,12}$ The percutaneous approach to caliceal diverticula is generally the most effective treatment. It can extract stones and relieve nephralgia. The approach can be to obliterate the cavity entirely or to open the neck and improve antegrade urinary drainage. Inability of the nephroscope to access the diverticular neck can be circumvented by creating a new opening. ${ }^{13}$ One group of authors ${ }^{14}$ reported a $93 \%$ stone-free rate and $76 \%$ infundibular-patency rate 2 years after percutaneous surgery, with major complications in $6.6 \%$ of patients and minor complications in another $13.4 \%$. While percutaneous procedures are associated with a high success rate, they risk urine leak, hematoma, and pneumothorax and necessitate at least overnight inpatient observation. Moreover, percutaneous nephrolithotomy has been associated with development of infundibular stenosis, which may necessitate additional treatment. ${ }^{15,16}$

Urology provides the opportunity to utilize normal anatomy to access organs of interest. Ureteroscopic manipulation of the renal collecting system avoids external incision, thereby limiting postoperative pain. Ureteroscopy has been successful for a variety of intrarenal pathologies. Ureteroscopic management of caliceal diverticula (alone or in combination with nephrolithiasis) can be successful in patients properly selected with regard to anatomy. Limitations on ureteroscope deflection and associated difficulty in passing a laser or other instrument through the working channel make lower-pole structures more difficult to 
treat. In the series of Baldwin and associates, ${ }^{11}$ the only caliceal diverticulum that could not be accessed ureteroscopically was in a lower pole; the rate of ureteroscopic success was $29 \%$ for lower-pole caliceal diverticula compared with $84 \%$ for upper-pole and central diverticula in the study of Batter and Dretler. ${ }^{17}$ Similarly, the only hydrocalix not accessed in our series was in the lower pole.

Our success rate for ureteroscopic treatment of hydrocalices was lower than the rates reported by other authors for the treatment of caliceal diverticula. Whereas all accessed diverticula were treated successfully in the series of Batter and Dretler, ${ }^{17}$ we did not have persistent success in all the hydrocalices that we were able to treat. Similar to the experience of Baldwin and associates, ${ }^{11}$ who reported that $50 \%$ of their patients were not rendered stone-free, we had a disappointing stone-free rate even when there was apparent good fragmentation of the stone at the time of surgery. Grasso et $\mathrm{al}^{12}$ reported on two successful ureteroscopic treatments of caliceal diverticula, but one patient required an additional percutaneous treatment. One experienced group concluded that percutaneous intervention for the treatment of symptomatic caliceal diverticula associated with stones was more effective than ureteroscopy with regard to stone burden and pain $(86 \% \vee 35 \%)$, but the hospital stay was shorter and the complication rate lower with ureteroscopy. ${ }^{18}$

In our series, four patients ultimately required percutaneous treatment; one patient had failed access at the initial procedure, and the remaining patients developed recurrent stenoses. In the latter three patients, repeat endoscopic management was attempted without success prior to creating percutaneous access. Although our series is small, our experience suggests that recurrently obstructed hydrocalices may not be amenable to retrograde endoscopic management.

Case series have inherent limitations, largely referable to the absence of a formal study design as well as to retrospective data review. This paper reports on 10 patients undergoing 12 procedures; while ureteroscopy was attempted in all patients, the choice of intervention to open the hydrocalix (e.g., laser, balloon dilatation, or cautery) was left to the discretion of the primary surgeon. Retrospectively, it is difficult to determine if particular characteristics of the hydrocalix prompted the surgeon to favor one intervention over another. Data such as the dimensions of the stricture, anatomic findings suggesting the presence of blood vessels at the planned surgical site, and the tissue quality at the time of surgery were not recorded, but such information may have been helpful to better elucidate the characteristics of hydrocalices amenable to ureteroscopic treatment. Although most complications of ureteroscopy are minor, serious or even life-threatening complications such as hemorrhage can occur. Because the inherent variations in hydrocaliceal anatomy may make both access and intervention technically challenging, patients should be counseled carefully on the risks of endoscopic treatment.

Finally, our follow-up is quite variable, largely reflecting the routine postoperative visit with imaging at 1 month. Only one patient was lost to follow-up, but this is significant, given the small size of our series. Moreover, of the other patients, follow-up imaging was not always performed at the 1-month visit. Most of our patients were referred to our institution from the community for specialized care and have resumed care with their regular urologists. The patients with the longest follow- ups either had complex medical conditions and had their routine urologic care managed at our institution (No. 1) or had a late recurrence of pain necessitating a second procedure (No. 6). Conversely, patients with the shortest follow-ups had treatment success by imaging and were referred back to their regular urologists for continued care. Nonetheless, it is impossible to know the long-term infundibular patency rates in patients without follow-up or who receive care in the community.

\section{CONCLUSION}

In our series, flexible ureteroscopy with endoscopic electrocautery, laser, or balloon dilatation or some combination was a safe means of managing symptomatic hydrocalices. Our series was similar to that of investigators reporting on ureteroscopic treatment of caliceal diverticula, in that symptomatic success was achieved in only about half the patients. In addition, the anatomy of the hydrocalix may limit passage of stone fragments. Nonetheless, we contend that the ureteroscopic approach spared some of these patients a more invasive percutaneous treatment. The presence of hydrocalix should not exclude otherwise-appropriate patients from an initial attempt at endoscopic management, especially when the overall benefits of endoscopy, including shorted convalescence and cost savings, are taken into consideration.

\section{REFERENCES}

1. Bodner DR, Caldamone AA, Resnick MI. Acquired infundibular stenosis. Urology 1987;29:19-21.

2. Husmann DA, Kramer SA, Malek RS, Allen TD. Infundibulopelvic stenosis: A long-term followup. J Urol 1994;152:837-840.

3. Hsu THS, Streem SB. Metabolic abnormalities in patients with caliceal diverticular calculi. J Urol 1998;160:1640-1642.

4. Lang EK. Percutaneous infundibuloplasty: Management of calyceal diverticula and infundibular stenosis. Radiology 1991;181: 871-877.

5. Bellman GC, Brock WA, Smith AD. Endourologic management of obstructed hydrocalix after blunt renal trauma. Urology 1994;43: 546-548.

6. Hwang TK, Park YH. Endoscopic infundibulotomy in tuberculous renal infundibular stricture. J Urol 1994;151:852-854.

7. Yip SK, Peh WC, Li JH, Cheung MC. Case report: Percutaneous balloon dilatation and ureteral stenting for tuberculous renal infundibular and ureteral strictures. Ann Acad Med Singapore 1999;28:284-287.

8. Kim HL, Gerber GS. Use of ureteroscopy and holmium: yttrium-aluminum-garnet laser in the treatment of an infundibular stenosis. Urology 2000;55:129-131.

9. Chong TW, Bui MH, Fuchs GJ. Calyceal diverticula: Ureteroscopic management. Urol Clin North Am 2000;27:647-654.

10. Kulkarni R, Wolf JS, Padiyar N, et al. Severe intrarenal fibrosis, infundibular stenosis, renal cysts, and persistent perilobar nephrogenic rests in a patient with Beckwith-Wiedemann syndrome 27 years after diffuse nephroblastomatosis and Wilms' tumor: Natural progression or a consequence of treatment? J Pediatr Hematol Oncol 2002;24:389-393.

11. Baldwin DD, Beaghler MA, Ruckle HC, et al. Ureteroscopic treatment of symptomatic caliceal diverticular calculi. Techn Urol 1998;4:92-98.

12. Grasso M, Lang G, Loisides P, et al. Endoscopic management of 
the symptomatic caliceal diverticular calculus. J Urol 1995;153: $1878-1881$.

13. Auge BK, Munver R, Jouramas J, et al. Neoinfundibulotomy for the management of symptomatic calyceal diverticula. $\mathrm{J}$ Urol 2002;167:1616-1620.

14. Shalhav AL, Soble JJ, Nakada SY, et al. Long-term outcome of caliceal diverticula following percutaneous endosurgical management. J Urol 1998;160:1635-1639.

15. Parsons JK, Jarrett TW, Lancini V, et al. Infundibular stenosis after percutaneous nephrolithotomy. J Urol 2002;167:35-38.

16. Weir MJ, Honey RJD'A. Complete infundibular obliteration following percutaneous nephrolithotomy. J Urol 1999;161:12741275.

17. Batter SJ, Dretler SP. Ureterorenoscopic approach to the symptomatic caliceal diverticulum. J Urol 1997;158:709-713.
18. Auge BK, Munver R, Kourambas J, Newman GE, Preminger GM. Endoscopic management of symptomatic calyceal diverticula: A retrospective comparison of percutaneous nephrolithotripsy and endoscopy. J Endourol 2002;16:557-563.

Address reprint requests to: J. Stuart Wolf, Jr., M.D.

Dept. of Urology

University of Michigan

1500 East Medical Center Drive, 3875 TC Ann Arbor, MI 48109-0330

E-mail:wolfs@umich.edu 
This article has been cited by:

1. K Kiryluk, R A Rabenou, E R Goldberg, M Gupta. 2008. The Case 7 Thirty-one-year old woman with hypertension and abnormal renal imaging. Kidney International 73:5, 659-660. [CrossRef]

2. James A. Kontak, Andrew D. Wright, Thomas M.T. Turk . 2007. Treatment of Symptomatic Caliceal Diverticula Using a Mini-Percutaneous Technique with Greater Than 3-Year Follow-UpTreatment of Symptomatic Caliceal Diverticula Using a Mini-Percutaneous Technique with Greater Than 3-Year Follow-Up. Journal of Endourology 21:8, 862-865. [Abstract] [PDF] [PDF Plus] 